Self review

\title{
Patterns of biological effects of electromagnetic terahertz waves at frequencies of active cellular metabolites of post stressed changes in hemostasis
}

\author{
Vyacheslav F. Kirichuk, Alexander A. Tsymbal
}

Saratov State Medical University n.a. V.I. Razumovsky, Saratov, Russia

Received 30 May 2013, Revised 15 June 2013, Accepted 23 August 2013

(C) 2013, Kirichuk V.F., Tsymbal A.A.

(C) 2013, Russian Open Medical Journal

Abstract: There had been studied the influence of electromagnetic waves at terahertz frequencies of active cellular metabolites (nitric oxide $150.176-150.664 \mathrm{GHz}$ and $129.0 \mathrm{GHz}$ atmospheric oxygen) on the changed parameters of homeostasis in experimental animals. It is shown that in the 15 minute exposure mode terahertz waves at frequencies of nitric oxide 150.176-150.664 GHz observed partial recovery of the changed parameters of homeostasis in stressed animals, and at 30 minute mode, the impact of these waves there is complete recovery of homeostatic parameters of male rats in a state of acute and prolonged immobilization stress.

Keywords: terahertz waves, immobilization stress, homeostasis, molecular spectra, nitric oxide, atmospheric oxygen

Cite as Kirichuk VF, Tsymbal AA. Patterns of biological effects of electromagnetic terahertz waves at frequencies of active cellular metabolites of post stressed changes in hemostasis. Russian Open Medical Journal 2013; 2: 0403.

Correspondence to Prof. Vyacheslav F. Kirichuk. Address: Department of normal physiology n.a. I.A. Chuevsky, Saratov State Medical University n.a. V.I. Razumovsky, 112, Bolshaya Kazachiya str., Saratov, 410012, Russia. E-mail: normalf@yandex.ru

\section{Introduction}

One of the current problems of physiology is studying of an organism and environment intercourses. Most of significant biotic factors of the environment are of the electromagnetic origin [1]. In particular electromagnetic irradiations are used as an information medium in biosphere. Man-made source of radiation of different bands also influence live organisms and they are widely used $[2,3]$.

Some of electromagnetic irradiation types are well known and used in industry, everyday life, clinical practice - infrared, ultraviolet (UV), ultrahigh (UHF), classical extremely high frequency irradiation [2, 3].

THz-band is a focus of attention of specialist not only of radionics sphere, but biomedical technologies as well. This is connected first with effects of the intercourse of this irradiation band and biological medias, and also with the fact that this is the band of molecular emission and absorption spectrum (MEAS) of the most significant cellular metabolites $\left(\mathrm{NO}, \mathrm{O}_{2}, \mathrm{CO}_{2}, \mathrm{CO}, \mathrm{OH}^{-}\right.$, etc.) $[4,5]$.

The fundamental base of functioning of complicated biological systems is molecules-metabolites, stable and strictly replicate molecular structures of biological media. So the determining control of their reaction ability with the help of irradiation (coincident with their MEAS) can directly influence metabolism process in media. This corresponds to the idea of a substance and field as a union system [4].

The study aimed to specify the laws and mechanisms of biological effect of electromagnetic terahertz waves at frequencies of active cellular metabolites on changes in hemostasis parameters in experimental animals in different stress models.

\section{Methods}

Experiments had been organized in white outbread male rats of $180-260 \mathrm{~g}$ (from the vivarium). There had been used 905 reproductive animals, 68 experimental groups had been formed (from 10 to 15 white male rats in each one). The rats had got a standard feeding once a day and had a free access to water. All of the rats had been in the same conditions during the experiment.

The rats management, acute and prolonged immobilization stresses modeling, and diversion of the animals from the experiment had been organized according to Geneva Convention "International Guiding Principles for Biomedical Research Involving Animals" (Geneva, 1990).

The model of immobilization stresses was the base of the research. There had been used the following experimental models:

i) acute immobilization stress was stimulated by rigid fixation of the rats in dorsal position by the limbs, using ligatures (single-shot, 3 hours).

ii) prolonged immobilization stress was stimulated by rigid fixation of the rats in dorsal position by the limbs, using ligatures (a 5-day course daily procedure, 3 hours).

For irradiation of the animals there had been used portable medical device for THz therapy "Orbita", developed in the Central research institute of measuring equipment (Saratov, Russia). There had been irradiation of $\mathrm{THz}$ band of nitric oxide 150.176$150.664 \mathrm{GHz}$ and $129.0 \mathrm{GHz}$ atmospheric oxygen frequencies. $3 \mathrm{~cm}^{2}$ skin areas above the metasternum had been irradiated. Irradiator had been located $1.5 \mathrm{~cm}$ above the body of an animal. The "NO" generator radiant power was $0.7 \mathrm{~mW}$, the power density that arrives at the $3 \mathrm{~cm}^{2}$ skin area was $0.2 \mathrm{~mW} / \mathrm{cm}^{2}$. Using 
the " $\mathrm{O}_{2}$ " generator radiant power density that arrives at the $3 \mathrm{~cm}^{2}$ skin area was $100 \mathrm{~mW} / \mathrm{cm}^{2}$. The duration of the single-shot irradiation or the 5-day course daily irradiation was 5, 15 и 30 minutes.

The blood samples had been taken into screened plastic testtubes with the help of a screened single-use syringe. If necessary there had been used $3.8 \%$ sodium citrate fluid as a blood anticoagulant in proportion of 9:1.

To estimate the function of a thyroid body in experimental animals there had been performed the following tests: quantitative determination of a concentration of free and bound thyroxin and triiodothyronine fractions in blood serum, concentration of thyroglobulin and thyrotropine, which are determined by an enzyme-linked immunosorbent assay using monoclonal antibodies with the help of reagents kit by Vector Best JSC (Russia). An analogical method had been used to measure the corticosterone concentration in blood serum of male rats.

Coagulative and fibrinolytic blood properties of white rats had been studied with the help of tests using turbidimetric coagulometer CGL 2110 "Solar» (Minsk, Belarus) and reagents by Scientific and Production Company «Renam» (Moscow, Russia). Determination of D-dimers in blood plasma had been organized by immunometric sandwich assay (NycoCard D-dimer using NycoCard Reader II).

The lipid peroxidation (LPO) processes in experimental animals was studied using standard methods of primary and secondary LPO products determination (lipid hydroperoxides, malondialdehyde): intracellular and in blood plasma. Together with this there had been measured erythrocytes peroxide resistance and proteolysis markers - molecules of an average mass in blood plasma. To study the condition of enzymatic and nonenzymatic components of blood antioxidant system there had been determined superoxide dismutase and catalase potency in erythrocytes, decrease of the amount of general sulfhydryl groups and vitamin $\mathrm{E}$ in blood serum.

The study of basic indices of metabolic status at experimental animals, lipid, (cholesterin, triglycerids), carbohydrate metabolism (blood glucose), indices of nitrogenous compound metabolism, transaminase (aspartate aminotransferase (AST) and alanine aminotransferase (ALT)), total activity of lactate dehydrogenase had been organized with the help of automatic biochemical analyzer Vitalab Flexor «E» (by Vital Scientific, Holland).

Determination of other indices of metabolic status (total protein level, albumins concentration in blood serum, potency on glutathione-S-transferase and ceruloplasmin) at laboratory animals was organized according to standard methods.

Blood gas and electrolytic blood composition: $\mathrm{pH}$ (value of active reaction of media); carbon dioxide tension $\left(\mathrm{pCO}_{2}\right)$; oxygen tension $\left(\mathrm{pO}_{2}\right)$; bicarbonate concentration $\left(\mathrm{HCO}_{3}\right)$; sodium ion $\left(\mathrm{Na}^{+}\right)$ and kalium $\left(\mathrm{K}^{+}\right)$had been measured by blood gas and electrolytes analyzer Rapidlab 348 (Bayer diagnostics, USA) at intact white male rats and in experimental groups.

Nitrites concentration in blood serum of experimental animals had been determined by V.B. Karpuk's modified method, there had been a reaction based on nitrites' ability to diazotize sulphanilic acid and to produce red-violet diaza compound with 1naphthylamine [6].

Statistical analysis of the results had been done with the help of Statistica 6.0 (Statsoft, USA).

\section{Results and Discussion \\ Changes in hypophysial-thiroid potency at stressed male rats under the effect of terahertz frequencies of active cellular metabolites [7-9]}

During the research there had been found out that at intact animals thyrotropine was $0.62 \mathrm{mlU} / \mathrm{L}$, at immobilized animals under the acute stress conditions it increased up to $1.20 \mathrm{mIU} / \mathrm{L}$ and up to $1.25 \mathrm{mIU} / \mathrm{L}$ - under the prolonged stress conditions.

At the rats under the acute immobilization stress there had been fixed functional activity depression of the thyroid body, which was clear from the statistically valid decrease of the concentration of free and bound thyroxin and triiodothyronine forms as well. There had been reducing the value of $T_{3} / T_{4}$ proportion, concentration of thyroglobulin had been statistically valid reduced.

On the background of the given change there had been statistically significant increase of the thyrotropine concentration.

The 5-minute irradiation of $\mathrm{THz}$-band electromagnetic waves of 150.176-150.664 GHz nitrogen oxide occurrence on animals under the acute immobilization stress does not cause much change in examined indices of hypophysial-thiroid potency.

During the 15-minute irradiation of $\mathrm{THz}$-band electromagnetic waves of nitrogen oxide occurrence on the background of the acute immobilization stress, there had been revealed partial normalization of hypophysial-thiroid potency. So there is statistically valid normalization of free triiodothyronine, nevertheless, all of the other indices of functional activity of hypophysis and thyroid body differ from the control group.

The 30-minute irradiation of the same occurrence under the acute stress causes total normalization of the thyroid body functional activity. At the same time the concentration of free and bound thyroxin and triiodothyronine forms, concentration of thyroglobulin, $T_{3} / T_{4}$ proportion and thyrotropine activity, and did not differ from the control group.

At the rats under prolonged acute immobilization stress there had been fixed the thyroid body functional activity depression, which had been appeared as a decrease of the concentration of free and bound thyroxin and triiodothyronine forms as well. The $T_{3} / T_{4}$ proportion value had been reduced.

At the male rats under the prolonged immobilization stress there had been reduced the concentration of thyroglobulin (statistically valid).

At the rats under the prolonged immobilization stress condition there had been fixed (more evident than after acute stress) the thyroid body functional activity depression, which had been appeared in decrease of the concentration of free and bound thyroxin and triiodothyronine forms as well. The $T_{3} / T_{4}$ proportion value had been reduced.

After a 5-day course irradiation of $\mathrm{THz}$-band electromagnetic waves of $150.176-150.664 \mathrm{GHz}$ nitrogen oxide occurrence on animals under the immobilization stress (for 5 minutes) there had not been fixed change in the thyroid body functional activity. This is evident from the absent of the difference between the basic studied parameters characterizing the thyroid body functions of this group comparing with the groups of animals under the prolonged stress. So the thyrotropine concentration after a 5-day course 5-minute irradiation of $150.176-150.664 \mathrm{GHz}$ nitrogen oxide occurrence had not been changed (statistically valid) - 1.19 $\mathrm{mIU} / \mathrm{L}$. The concentration of free and bound thyroxin and triiodothyronine forms had not also been normalized, and the 
$T_{3} / T_{4}$ proportion value had been differed from the same parameter of intact animals. The concentration of thyroglobulin had not been changed (statistically significant) after a 5-day course 5-minute irradiation of $150.176-150.664 \mathrm{GHz}$ nitrogen oxide occurrence; it was $0.62 \mathrm{ng} / \mathrm{l}$.

After a 5-day course 15-minute irradiation of THz-band electromagnetic waves of nitrogen oxide occurrence on the background of the prolonged immobilization stress, there had been revealed partial normalization of the thyroid body functional activity. So the given irradiation mode promotes total normalization of thyroglobulin concentration, but all of the other parameters of the thyroid body activity had been differed from the control group (statistically valid).

After a 5-day course 30-minute irradiation of 150.176$150.664 \mathrm{GHz}$ nitrogen oxide occurrence (under prolong immobilization) there had been revealed total normalization of the thyroid body functional activity. The concentration of free and bound thyroxin and triiodothyronine forms had not also been normalized; thyrotropine activity and the $T_{3} / T_{4}$ proportion value had not been differed from the same parameter of the control group.

So the 30-minute mode of irradiation on the background of acute and immobilization stress of the male rats had been the most effective for the normalization of hypophysial-thiroid potency at experimental animals.

The effect of terahertz frequencies of molecular spectrum of $129.0 \mathrm{GHz}$ atmospheric oxygen occurrence on corticosterone concentration - the stress-reaction marker [10]

To estimate an ability of $\mathrm{THz}$-band electromagnetic waves of $129.0 \mathrm{GHz}$ atmospheric oxygen to limit (modulate) the course of stress-reaction there had been organized set of experiments, and there had been studied the dynamics of corticosterone concentration (the stress-reaction marker) at irradiated white male rats on the background of acute and prolonged immobilization stresses.

After the performed experiments we had found out that corticosterone concentration at intact animals was $62.2 \mathrm{nM} / \mathrm{l}$. It was indicated that at rats under the acute and prolonged immobilization stresses the functional activity of adrenal cortex increased. This had been accompanied by a statistically valid corticosterone concentration increase in blood serum (comparing with the control group). So, under the acute stress conditions it increases almost in 2.5 times up to $175.0 \mathrm{nM} / \mathrm{l}$, under the prolonged stress - in 3.5 times up to $200.0 \mathrm{nM} / \mathrm{l}$.

The 5-minute irradiation of $\mathrm{THz}$-band electromagnetic waves of $129.0 \mathrm{GHz}$ atmospheric oxygen caused (on the background of acute stress) statistically valid decrease of corticosterone concentration to $102.0 \mathrm{nM} / \mathrm{l}$. Though, the concentration of corticosterone had not reduced to parameters of intact animals, which is evident from statistically valid differences between intact animals and the group of animals after the 5-minute irradiation.

The usage of the 15-minute $\mathrm{THz}$-band electromagnetic waves of $129.0 \mathrm{GHz}$ atmospheric oxygen to limit in acute stress case displayed total restoration of corticosterone concentration level in blood serum at the experimental animals. So the concentration of the studied hormone had fallen by half after the irradiation and got $71.1 \mathrm{nM} / \mathrm{l}$.

The results of the experiments with 30-minute THz-band electromagnetic waves of $129.0 \mathrm{GHz}$ atmospheric oxygen (on the background of an acute immobilization) show the restoration of corticosterone concentration (statistically valid) up to $68.3 \mathrm{nM} / \mathrm{l}$. The analysis of the results had not been shown any differences in the studied hormone concentration in the given group of animals and intact white male rats.

On the background of the prolonged immobilization stress a 5day course exposure of $\mathrm{THz}$-band electromagnetic waves of 129.0 $\mathrm{GHz}$ atmospheric oxygen had not caused change in glucocorticoid concentration in blood serum (corticosterone as well). This is evident from the statistically valid differences in the studied parameter (comparing with the parameters in the group of control). So, after the irradiation, the corticosterone concentration was $212.9 \mathrm{nM} / \mathrm{l}$.

A 5-day course 15-minute irradiation of THz-band electromagnetic waves of $129.0 \mathrm{GHz}$ atmospheric oxygen on the background of the prolonged immobilization stress leads to an incomplete restoration of the corticosterone concentration. After the irradiation the level of the studied hormone decreased to $141.7 \mathrm{nM} / \mathrm{l}$, but there had been kept statistically valid differences between intact animals and animals after the 15-minute irradiation.

A 5-day course 30-minute irradiation of $129.0 \mathrm{GHz}$ atmospheric oxygen (on the background of the prolonged immobilization) is accompanied by the total normalization of the corticosterone concentration, which is evident from the absence of the statistically valid differences comparing with intact animals. In this irradiation time mode the corticosterone concentration was $73.0 \mathrm{nM} / \mathrm{l}$.

Now it can be seen that there had been revealed the normalizing effect of $\mathrm{THz}$-band electromagnetic waves of $129.0 \mathrm{GHz}$ atmospheric oxygen influencing the corticosterone concentration in blood of male rats. The most effective time modes were the 15-minute irradiation on the background of an acute stress and a5-day course 30-minute irradiation on the background of the prolonged immobilization of the animals.

THz-band electromagnetic waves of $129.0 \mathrm{GHz}$ atmospheric oxygen in normalization of fibrinolysis and hemostasis system parameters in experimental animals $[11,12]$

The results of the study show that there are significant hypercoagulative changes in animals under an acute immobilization stress, they show as a reduction of activated partial thromboplastin time (APTT), prothrombin time, international normalized ratio (INR) reduction; simultaneous activation of coagulation phase III, as thrombin time had a direct tendency to reduction, and fibrinogen concentration and XIII factor activity are increased. At the same time anticoagulant blood potency reduced. So protein $\mathrm{C}$ activity at rats under the acute stress decreased from 0.87 c.u. to 0.54 c.u.

At the male rats under acute immobilization there had been fixed blood fibrinolytic activity depression, which is shown as prolonged time of euglobulin clutter lysis from 14 minutes to 24.0 minutes, and the time induced by streptokinase euglobulin fibrinolysis from $130.0 \mathrm{sec}$. to $180.0 \mathrm{sec}$. The index of plasminogen reserve statistically valid reduced from $70.1 \%$ to $50.5 \%$.

During the development of an acute stress-reaction in experimental animals, there had not been found statistically valid change in D-dimer concentration, solvable fibrin-monomeric complexes and fibrinogen degradation products. 
The 5-minute irradiation of $\mathrm{THz}$-band electromagnetic waves of $129.0 \mathrm{GHz}$ atmospheric oxygen had not caused (on the background of acute immobilization stress) significant change in defected parameters of hemostasis and fibrinolysis, which is confirmed by the absence of statistically valid differences in parameters characterizing coagulative cascade and fibrinolysis (comparing with the stressed animals). Though, there is a statistically valid difference between parameters of a coagulative component in hemostasis system and blood fibrinolytic activity (comparing with the intact animals).

It had been revealed that a 15-minute irradiation of THz-band electromagnetic waves of $129.0 \mathrm{GHz}$ atmospheric oxygen (in an acute stress) caused partial normalization of indices characterizing coagulative cascade and fibrinolysis, because APTT and thrombin time had been totally normalized and did not differ from the intact animals. Though all the other hemostasis indices differed from the indices of the intact animals (statistically valid).

30-minutes irradiation of the THz-band electromagnetic waves of $129.0 \mathrm{GHz}$ atmospheric oxygen totally normalize hemocoagulation and fibrinolysis processes, as all of the studied indices coagulative component in hemostasis system and blood fibrinolysis did not differ from indices of the intact animals (statistically valid).

During the study of a coagulative component in hemostasis system and blood fibrinolytic activity in the rats under the prolonged immobilization, there had been revealed (statistically valid) significant differences comparing with the control group: prolonged APTT, prolonged prothrombin time and INR, which shows a developing deficit of blood and tissue prothrombinase. Activity of coagulation phase III is reduced, as thrombin time had a direct tendency to prolongation, which is caused by hypofibrinogenemia and excessive concentration of degradation products of fibrin in blood plasma (these products are antithrombine). There had been decrease of fibrin stabilizing factor activity.

Prolonged immobilization stress in male rats had been accompanied by blood fibrinolytic and anticoagulant activity. Protein C level decreased to 0.42 c.u. Hageman-factor-dependent fibrinolysis had been activated, which was accompanied by shortening of euglobulin lysis from $14 \mathrm{~min}$ to $11 \mathrm{~min}$, and there had been accelerated induced by streptokinase euglobulin fibrinolysis from $130 \mathrm{sec}$. to $120 \mathrm{sec}$., and index of plasminogen reserve statistically valid increased $72.8 \%$.

In prolonged immobilization stress at male rats there had been found an increase of D-dimer concentration to $0,5 \mathrm{mg} / \mathrm{l}$, fibrinmonomeric complexes and fibrinogen degradation products typical to intravascular thrombosis.

A 5-day course 5-minute irradiation of the given frequencies occurrence in animals under the prolonged stress had not revealed changes in the studied parameters of a coagulative component in hemostasis and fibrinolysis.

A 5-day course 15-minute irradiation the $\mathrm{THz}$-band electromagnetic waves of $129.0 \mathrm{GHz}$ atmospheric oxygen had led to the partial normalization of changed parameters of a coagulative component in hemostasis system, anticoagulant blood activity and fibrinolytic blood properties. The APTT, thrombin time and induced by streptokinase euglobulin fibrinolysis had been totally normalized and did not differ (statistically valid) from the parameters of the intact group of animals. But all of the other studied parameters of hemostasis and fibrinolysis in experimental animals differed from the parameters of the intact animals.
A 5-day course 30-minute irradiation of $129.0 \mathrm{GHz}$ atmospheric oxygen causes total normalization of changed parameters of clotting and fibrinolysis, as all of the other studied parameters of a coagulative component in hemostasis system, anticoagulant blood potency and its fibrinolytic potency did not differ (statistically valid) from the parameters of the intact animals.

With reference to the given results, we can conclude that there was a positive effect on changed blood coagulation properties and fibrinolysis processes in stressed animals. Daily 30minute irradiation was the most effective in acute and prolonged stresses in normalization of the clotting and fibrinolytic potency.

Effect of THz radiation of 150.176-150.664 GHz nitrogen oxide occurrence on blood antioxidant potency and lipid peroxidation process at stressed rats [13-15]

It is displayed that in rats under the immobilization stress the process of lipid peroxidation (LPO) had been extremely activated. This is followed by an increase (statistically valid comparing with the control group) of toxical lipid peroxidation intermediate products - lipid hydroperoxides, malondialdehyde.

Overconcentration of the LPO products at immobilization stress was followed by cytolysis syndrome progressing. This was clear from the overconcentration of the molecules of an average mass in blood.

Revealed fact of an overconcentration of lipid peroxidation intermediate products in blood under the immobilization stress condition is caused by the defect of functional activity of enzymatic and nonenzymatic components. This is the result of the reduction of the following indices: superoxide dismutase (SOD) and catalase potency, general sulfhydryl groups (SH-groups) in blood serum and erythrocytes peroxide resistance comparing with the control group.

The 5-minute irradiation of THz-band electromagnetic waves of $150.176-150.664 \mathrm{GHz}$ nitrogen oxide occurrence on animals under the acute immobilization stress does not cause much change of blood antioxidant system or in lipid peroxidation.

During the 15-minute irradiation of $\mathrm{THz}$-band electromagnetic waves of nitrogen oxide occurrence on the background of the immobilization stress, there had been revealed partial (but more evident than during the 5-minute irradiation) normalization of lipid peroxidation process and antioxidant activity. This is clear from the reduction of concentration of toxical intermediate products of lipid peroxidation, and partial recovery of blood antioxidant properties.

The 30-minute irradiation of the same occurrence causes total normalization of lipid peroxidation processes, which is evident from the decrease of concentration of toxical intermediate products of lipid peroxidation as it is at intact animals. Functional activity of enzymatic and nonenzymatic components in antioxidant cell reaction had also been recovered and does not differ (statistically valid) from the indices of intact animals.

At male rats under prolonged immobilization stress (comparing with the animals under acute immobilization) there had been analogical but more evident changes in studied LPO indices. Appearance of LPO products correlated with autointoxication developing, which is clear from the overconcentration of of the molecules of an average mass in blood. There had been fixed depression of enzymatic and nonenzymatic components in antioxidant system in rats under the prolonged stress. This came out in statistically valid decrease of superoxide dismutase (SOD) and catalase potency in erythrocytes, 
decrease of the amount of general sulfhydryl groups (SH-groups) and vitamin $\mathrm{E}$ in blood serum.

Daily 5-minute irradiation of $\mathrm{THz}$-band electromagnetic waves of $150.176-150.664 \mathrm{GHz}$ nitrogen oxide occurrence on animals under the immobilization stress does not cause much change of blood antioxidant system or in lipid peroxidation.

It was displayed that during the daily 15-minute irradiation of THz-band electromagnetic waves of nitrogen oxide occurrence on the background of the immobilization stress, there had been revealed partial normalization of lipid peroxidation process and antioxidant activity. This is clear from the reduction of concentration of toxical intermediate products of lipid peroxidation - malondialdehyde and lipid hydroperoxides and partial normalization of antioxidant blood properties.

Daily 30-minute irradiation of the same occurrence causes total normalization of lipid peroxidation processes, which is evident from the decrease of concentration of toxical intermediate products of lipid peroxidation as it is at intact animals. So the given irradiation mode promotes reactivation of enzymatic and nonenzymatic components of blood antioxidant system, which appeared in normalization of superoxide dismutase (SOD) and catalase potency, increase of the amount of general sulfhydryl groups (SH-groups) and vitamin E in blood serum (as in control group).

So the irradiation of $\mathrm{THz}$-band electromagnetic waves of 150.176-150.664 GHz nitrogen oxide occurrence partially or totally depresses the intensification of lipid peroxidation processes, decreases a cytolysis appearance, recovers the condition of components in antioxidant system (depending on an experiment conditions and the time of the irradiation).

Use of $\mathrm{THz}$ radiation of 150.176-150.664 GHz nitrogen oxide occurrence in correction of post-stress change in blood gases and electrolytes of blood at male rats [16-18]

We had found changes in blood gases at rats under the acute stress. This is appeared in decrease of carbon dioxide tension (statistically valid), evident decrease of oxygen tension, bicarbonate concentration and $\mathrm{pH}$ media reduction.

In this group of animals there had been also changed electrolytes of blood, which is evident from an increase of kalium ions concentration (statistically valid). An acute immobilization does not affect much the sodium ions (statistically valid).

The 5-minute irradiation of $\mathrm{THz}$-band electromagnetic waves of $150.176-150.664 \mathrm{GHz}$ nitrogen oxide occurrence on animals under the acute immobilization stress does not cause much change in the studied parameters of blood gases and electrolytes of blood.

During the 15-minute irradiation of $\mathrm{THz}$-band electromagnetic waves of nitrogen oxide occurrence on the background of the acute immobilization stress, there had been revealed partial normalization of the parameters of blood gases and electrolytes of blood. This is evident from the normalization of carbon dioxide tension (statistically valid) and an increase of oxygen concentration in blood. Comparing with the stressed animals (statistically valid) there was a normalization of kalium ions concentration in blood. THz-band electromagnetic waves of nitrogen oxide occurrence does not affect significantly all the other studied parameters (in the given time mode).
The 30-minute irradiation of the same occurrence of the rats (on the background of the acute immobilization stress) causes total normalization of blood gases and blood electrolytes. At the same time all of the studied parameters do not differ from the control group parameters (statistically valid). The oxygen tension in blood after the 30-minute irradiation of THz-band electromagnetic waves of $150.176-150.664 \mathrm{GHz}$ nitrogen oxide occurrence was higher than in the control group.

Changes in blood gases and electrolytes of blood were more significant in rats under the prolonged immobilization than in rats under the acute immobilization stress. This is appeared in decrease of carbon dioxide tension and oxygen tension, bicarbonate concentration and $\mathrm{pH}$ media reduction. There is more significant statistically valid increase of kalium ions concentration in blood, though there is no change in potential-producing sodium ions concentration.

A 5-day course 5-minute irradiation of $\mathrm{THz}$-band electromagnetic waves of $150.176-150.664 \mathrm{GHz}$ nitrogen oxide occurrence (under the prolonged immobilization stress) does not cause any changes in parameters of blood gases and electrolytes of blood.

The 5-day course 15-minute irradiation of the same occurrence of the rats (on the background of the prolonged immobilization stress) causes partial normalization of oxygenation and electrolytes of blood: there is a statistically valid, comparing with the prolong immobilization, normalization of carbon dioxide tension and oxygen tension, bicarbonate concentration and kalium ions concentration. At the same time, during the given irradiation mode, there was no evident differences between the level of oxygen tension, $\mathrm{pH}$ media and sodium ions concentration comparing with the group of animals under the prolonged immobilization stress.

A 5-day course 30-minute irradiation of THz-band electromagnetic waves of $150.176-150.664 \mathrm{GHz}$ nitrogen oxide occurrence on the background of the prolonged immobilization stress is characterized by the total normalization of blood gases and electrolytes of blood. All the other studied parameters don not differ from the parameters of the control group.

So 30-minute irradiation of $\mathrm{THz}$-band electromagnetic waves of $150.176-150.664 \mathrm{GHz}$ nitrogen oxide occurrence is the most effective mode in normalization of blood gases and electrolytes of blood (both under the prolonged and immobilization stresses). 15minute irradiation of the same occurrence causes partial normalization of oxygenation and electrolytes of blood; 5-minute irradiation is not effective.

Normalization of the basic parameters of the metabolic status of stressed rats by THz-band electromagnetic waves of 150.176-150.664 GHz nitrogen oxide occurrence $[19,20]$

There had been fixed statistically valid changes in metabolic status in male rats under the stress (comparing with the group of intact animals). This was reflected in the fact that there was an increase of glucose and total concentration of protein in blood. At the same time, there had been changed a qualitative composition of blood protein spectrum: albumins level statistically valid reduced and percentage concentration of globulin fraction proteins had been increasing. There had been fixed an increase of total cholesterol, triglycerids, total lactate dehydrogenase (LDH) potency, transaminase level (ALT, AST), and increase of urea and creatinine in blood. Glutathione-S-transferase in male rats under 
the acute stress had also been statistically valid increased, which may be connected with assident ferment synthesis as a reaction on the post-stress homeostsasis change. There was in increase of ceruleoplasmin concentration in blood, which we consider as a compensatory reaction of an organism directed on an enzymic oxidation of biogenic amines (catecholamines) and other biologically active substances and stress-mediators.

A 5-minute irradiation of $\mathrm{THz}$-band electromagnetic waves of 150.176-150.664 GHz nitrogen oxide occurrence (under the acute stress) does not cause normalization of metabolic status parameters.

A 15-minute irradiation of the same occurrence of the rats (on the background of the prolonged immobilization stress) causes partial normalization of metabolic status parameters. Thus there had been statistically valid normalized and did not differ from the parameters of intact animals: triglycerids concentration, AST potency and glutathione-S-transferase.

A 30-minute irradiation of $\mathrm{THz}$-band electromagnetic waves of 150.176-150.664 GHz nitrogen oxide occurrence caused a total normalization of post-stress changes of metabolic status in experimental animals. There was a total recovery of the following parameters and they did not differ from the control group (statistically valid): carbohydrate metabolism, lipid metabolism, protein blood spectrum, the basic ferments potency.

Under the prolonged stress conditions there are more evident changes in the basic characteristics of metabolic status, than in acute immobilization. This is shown in more significant glucose concentration increase, total protein concentration decrease: albumin, dysproteinemia, cholesterol and triglycerids level increase, and urea and creatinine in blood. Serumal transaminase concentration (AST, ALT) and total LDH potency had increased more significantly than in acute stress. Under the prolonged stress conditions ceruleoplasmin concentration and glutathione-Stransferase potency had also been changed more than in acute immobilization stress.

It had been revealed that in a 5-minute irradiation of $\mathrm{THz}$-band electromagnetic waves of $150.176-150.664 \mathrm{GHz}$ nitrogen oxide occurrence (on the background of a prolonged immobilization stress) does not cause normalization of metabolic status parameters.

A 5-day course 15-minute irradiation of the same occurrence of the rats (on the background of the prolonged immobilization stress) causes partial normalization of metabolic status parameters. Only triglycerids level is recovered (statistically valid), while all of the other studied metabolic parameters differed from the parameters of the control group (statistically valid).

A 5-day course 30-minute irradiation of the same occurrence of the rats (on the background of the prolonged immobilization stress) causes total normalization of metabolic status parameters. At the same time the parameters of carbohydrate and lipid metabolism, nitrogenous compound metabolism, protein blood spectrum, serumal transaminase (AST, ALT) and LDH had been totally normalized and statistically valid did not differ from the control group. The presented results show that this irradiation mode causes total normalization of metabolic potency in white male rats under the prolonged stress.

So, on the ground of the presented results of the study, we can conclude that there is a positive effect of THz-band electromagnetic waves of $150.176-150.664 \mathrm{GHz}$ nitrogen oxide occurrence on the parameters of the metabolic status of experimental animals under the acute and prolonged immobilization stress conditions. The 30-minute irradiation time mode is the most effective in correction of the studied parameters. The 15-minute irradiation time mode on the background of the acute and prolonged immobilization stress conditions characterizing metabolism, had a partial effect, and there is no change in a 5-minute irradiation time mode.

The effect of THz-band electromagnetic waves of $129.0 \mathrm{GHz}$ atmospheric oxygen on stable nitrogen oxide metabolites concentration under stress conditions

Today there had been formulated an idea of a system of nitrogen oxide generation as of an isolated stress-limiting system [21].

To estimate nitrogen oxide synthesis activity, together with the direct measurement of the NO synthase (NOS) activity (with citrulline production), there is used stable metabolites concentration - nitrites and nitrates. The nitrites concentration is an objective criterion of a nitroxidergic system [21].

After the results analyzing there had been shown that the nitrites concentration in blood serum in intact male rats was $0.76 \mu / \mathrm{ml}$. During the acute stress-reaction development there had been fixed the reduction of the nitrites concentration in blood serum to $0.21 \mu / \mathrm{ml}$.

The reduction of a nitrogen oxide synthesis and, as a result, of a nitrites concentration in blood serum in acute stress immobilization stress is connected with an inhibitory action of stress-realizing hormones overconcentration, glucocorticosteroids and catecholamines as well [21].

Exposure of the animals under the acute stress with the $\mathrm{THz}$ band electromagnetic waves of $129.0 \mathrm{GHz}$ atmospheric oxygen (5minute irradiation) had led to a partial normalization of a nitrites concentration in blood serum to $0.35 \mu / \mathrm{ml}$.

A 15-minute irradiation of the $\mathrm{THz}$-band electromagnetic waves of $129.0 \mathrm{GHz}$ atmospheric oxygen (on the background of the acute stress) causes to an increase of a nitrites concentration in blood serum to $0.62 \mu / \mathrm{ml}$., this means a total normalization of a studied metabolite.

A 30-minute irradiation of the same occurrence of the rats (on the background of the acute stress) causes a total normalization of nitrogen oxide producing, because a nitrites concentration in blood serum was $0,82 \mu / \mathrm{ml}$.

In male rats under prolonged stress conditions there had been fixed the reduction of nitrites concentration in blood serum to $0.19 \mu / \mathrm{ml}$. So, under the prolonged immobilization stress there is more significant decrease of nitrogen oxide synthesis. This displays a decompensation of a stress-limiting system [21].

A 5-day course 5-minute irradiation of the given occurrence (under prolonged stress conditions) had not caused normalization of a nitrites concentration in blood serum in male rats; it was $0.22 \mu / \mathrm{ml}$.

A 5-day course 15-minute irradiation of the same occurrence (under prolonged stress conditions) had caused only partial normalization of a nitrites concentration in the studied blood samples in male rats; it was $0.50 \mu / \mathrm{ml}$.

A 5-day course 30-minute irradiation of the same occurrence under the prolonged immobilization had caused a total normalization of nitrogen oxide products in the studied blood samples. This is evident from the absence of statistically valid 
differences of in nitrites concentration in blood serum in intact and irradiated animals.

So, the most effective irradiation time mode in normalization of a nitrites concentration in rats is a 15-minute irradiation (in acute immobilization stress). Under the prolonged immobilization the 30-minute irradiation of the $\mathrm{THz}$-band electromagnetic waves of $129.0 \mathrm{GHz}$ atmospheric oxygen was effective.

The effect of THz-band electromagnetic waves of $129.0 \mathrm{GHz}$ atmospheric oxygen on nitrites concentration in blood under the acute stress conditions on the background of the intake of a nonselective inhibitor of constitutive NOS isoforms [23]

To identify the mechanisms of positive effects of the THz-band electromagnetic waves of $129.0 \mathrm{GHz}$ atmospheric oxygen on nitrites concentration in blood (under the acute stress conditions) there had been used a nonselective inhibitor of constitutive NOS isoforms L-NAME - NG-nitro-L-argenine methyl ether (Sigma, USA), which had been injected intramusculary in $25 \mathrm{mg} / \mathrm{kg}$ [22].

There had been fixed that the 15-minute and the 30-minute irradiation of rats under the acute stress using the $\mathrm{THz}$-band electromagnetic waves of $129.0 \mathrm{GHz}$ atmospheric oxygen on the background of the intake of a nonselective inhibitor of constitutive NOS isoforms had not caused a normalization of a nitrites concentration in blood serum. This is evident from statistically valid differences between nitrites concentrations of the control animals and irradiated animals (on the background of the intake of L-NAME).

Thus, an injection of a nonselective inhibitor of constitutive NOS isoforms in animals under the acute stress block normalizing effect of the THz-band electromagnetic waves of $129.0 \mathrm{GHz}$ atmospheric oxygen on a nitrites concentration in blood serum.

\section{Conflict of interest: none declared.}

\section{Reference}

1. Betsky OV, Kozmin AS, Yaremenko UG. Possible methods of terahertz waves usage. Biomedical Radioelectronics 2008; (3): 48-54. [Article in Russian]

2. Betsky OV, Krenitsky AP, Maiborodin AV. Biophysical effects of THzband and perspectives of progress in a new direction in biomedical technologies. Biomedical Radioelectronics 2003; (12): 3-6. [Article in Russian]

3. Kirichuk VF. Saratov medical scientists about physiological effects of electromagnetic waves of EHF- and THz-band. Biomedical Radioelectronics 2007; (2-4): 98-126. [Article in Russian]

4. Rothman LS, Barbe A, Benner CD, Brown LR, Camy-Peyret C, Carleer $M R$, et al. The HITRAN molecular spectroscopic database: edition of 2000 including updates through 2001. Journal of Quantitative Spectroscopy \& Radiative Transfer 2003; 82: 5-44. (doi: 10.1016/S0022-4073(03)00146-8).

5. Ignarro LG, Murad F. Nitric oxide: biochemistry, molecular biology and therapeutic implication. Adv Pharmacol 1995; 34.

6. Karpuk VB, Chernyak US, Shubich MG. Laboratorial monitoring of nitroxidergic vasorelaxation in subarachnoid hemorrhage. Clinical Laboratory and Diagnostics 2000; (5): 16-19. [Article in Russian]

7. Kirichuk VF, Tsymbal AA. Normalization of functional activity of thyroid gland with terahertz radiation at nitrogen oxide frequences in the conditions of experimental stress. Bulletin of New Medical Technologies 2009; 16(3): 111-113. [Article in Russian]

8. Kirichuk VF, Tsymbal AA. Use of terahertz electromagnetic radiation at nitric oxide frequencies for the correction of thyroid functional state during stress. Vestn Ross Akad Med Nauk (Annals of the Russian Academy of Medical Sciences) 2010; (4): 37-40. (PMID: 20540354)

9. Tsymbal AA, Kirichuk VF, Kurtukova MO. Influence of chronic stress and terahertz radiation at nitric oxide frequency on functional activity of thyroid gland. Saratov Journal of Medical Scientific Research 2010; 6(4): 767-771. [Article in Russian]

10. Tsymbal AA, Kirichuk VF, Antipova ON, Kurturova MO, Andronov EV. Changes in the level of corticosterone in the blood of experimental animals exposed to terahertz waves at a frequency of atmospheric oxygen $129.0 \mathrm{GHz}$ on a background of acute and prolonged stress. Biomedical Radioelectronics 2011; (8): 23-29. [Article in Russian]

11. Kirichuk VF, Tsymbal AA, Kreniskiy AP, Mayborodin AV. Theragerz radiations application on frequency of atmospheric oxygen of 129.0 $\mathrm{GHz}$ for correction coagulation hemostasis and fibrinolysis frustrations. Biomedical Radioelectronics 2009; (9): 11-17. [Article in Russian]

12. Kirichuk VF, Tsymbal AA. Use of Terahertz Electromagnetic Waves for Correcting Hemostasis Functions. Biomedical Engineering 2010; 44 (1): 11-14. (doi:10.1007/s10527-010-9145-1)

13. Kirichuk VF, Cymbal AA. Method for correcting the intensification of free radical oxidation in experimental stress. Bulletin of New Medical Technologies 2009; 16(1): 41-43. [Article in Russian]

14. Kirichuk VF, Tsymbal AA. Effects of terahertz irradiation at nitric oxide frequencies on intensity of lipoperoxidation and antioxidant properties of the blood under stress conditions. Bull Exp Biol Med 2009; 148(2): 200-203. (doi: 10.1007/s10517-009-0669-x)

15. Kirichuk BF, Tsymbal AA. Terahertz radiations application of nitrogen oxide frequencies for correction of antioxidant properties of blood and lipid peroxidation in the conditions of stress. Ross Fiziol Zh Im I M Sechenova 2010; 96(2): 121-127. (PMID: 20432719) [Article in Russian]

16. Kirichuk VF, Tsymbal AA. Peculiarities of the effect of terahertz radiation at nitric oxide frequencies on gaseous and electrolyte composition of the blood in different types of stress. Bull Exp Biol Med 2010; 150(2): 191-194. (PMID: 21240370)

17. Tsymbal AA, Kirichuk VF, Antipova ON, Andronov EV, Krenitsky AP. The dynamics of blood oxygenation in experimental animals exposed to terahertz waves at frequencies of nitric oxide in stress. Biomedical Radioelectronics 2011; (1): 36-39. [Article in Russian]

18. Tsymbal AA, Kirichuk VF. Changes gas and electrolyte structure of blood under influence terahertz radiations on frequencies nitrogen oxide $150,176-150,664 \mathrm{GHz}$ in the conditions of stress. Patol Fiziol Eksp Ter 2011; (1): 49-51. (PMID: 21688667)

19. Tsymbal AA, Kirichuk VF, Krenitsky AP, Betsky OV. Restoration of the main indicators of the metabolic status of terahertz waves at frequencies of nitric oxide $150.176 \ldots 150.664 \mathrm{GHz}$ in the experiment. Biomedical Radioelectronics 2011; (1): 30-35. [Article in Russian]

20. Tsymbal AA. Changes in protein spectrum of blood, the activity of glutathione-S-transferase, glucose and ceruloplasmin under the influence of terahertz waves at frequencies of nitric oxide 150.176...150.664 GHz with acute stress. Biomedical Radioelectronics 2011; (8): 30-35. [Article in Russian]

21. Manukhina EB, Malyshev IU. Nitrogen oxide stress-limiting system. Russian Physiological Journal n.a. I.M. Sechenov 2000; 86(10): 12831292. [Article in Russian]

22. Golikov PP. Nitrogen oxide in emergency cases clinic. Medpraktika Publ., Moscow, Russia, 2004. [Book in Russian]

23. Kirichuk VF, Tsymbal AA. Effects of terahertz radiation at atmospheric oxygen frequency of $129 \mathrm{GHz}$ on blood nitrite concentrations under conditions of different types of stress against the background of administration of nonselective inhibitor of constitutive NO-synthases. Bull Exp Biol Med 2012; 152(4): 435-438. (doi: 10.1007/s10517-0121547-5)

\section{Authors:}

Vyacheslav F. Kirichuk - MD, D.Sc. Professor, Honored Scientist of Russia, Head of Department of Normal Physiology n.a. I.A. Chuevsky, Saratov State Medical University n.a. V.I. Razumovsky, Saratov, Russia; 
Alexander A. Tsymbal - MD, PhD, Associate Professor, Department of Normal Physiology n.a. I.A. Chuevsky, Saratov State Medical University n.a.

V.I. Razumovsky, Saratov, Russia. 